\title{
Anomalies of Heat Capacity and Phase Transitions in $\operatorname{Tm}_{1-x} \mathrm{Yb}_{x} \mathrm{~B}_{12}$
}

\author{
N.E. Sluchanko ${ }^{a *}$, A.N. Azarevich ${ }^{a}$, A.V. Bogach ${ }^{a}$, V.V. Glushkov ${ }^{a}$, S.V. Demishev ${ }^{a}$, \\ S.Yu. Gavrilkin ${ }^{b}$, N.Yu. Shitsevalova ${ }^{c}$, V.B. Fillipov ${ }^{c}$, S. Gabani $^{d}$ And K. Flachbart ${ }^{d}$ \\ ${ }^{a}$ A.M. Prokhorov General Physics Institute of RAS, 38, Vavilov Str., Moscow, 119991, Russia \\ ${ }^{b}$ P.N. Lebedev Physical Institute of RAS, 53, Leniskii prospect, Moscow, 119991, Russia \\ ${ }^{c}$ Institute for Problems of Materials Science of UAS, 3, Krzhyzhanovsky Str., 03680 Kiev, Ukraine \\ ${ }^{d}$ Centre of Low Temperature Physics, IEP SAS and IPS FS UPJS, 04001 Košice, Slovakia
}

\begin{abstract}
In the system $\operatorname{Tm}_{1-x} \mathrm{Yb}_{x} \mathrm{~B}_{12}$ the specific heat has been studied in a wide range of $\mathrm{Yb}$-concentration in the vicinity of the quantum critical point $x_{\mathrm{C}} \approx 0.3$. The results were obtained on high quality single crystalline samples of $\mathrm{Tm}_{0.7} \mathrm{Yb}_{0.3} \mathrm{~B}_{12}$ compound placed near quantum critical point, both for antiferromagnetic metals $\left(x<x_{\mathrm{C}}\right)$ as well as for paramagnetic insulators $\left(x>x_{\mathrm{C}}\right)$ within a wide temperature range of 1.9-300 K in magnetic field up to $9 \mathrm{~T}$. The temperature dependence of the magnetic contribution to specific heat for $\mathrm{Tm}_{0.74} \mathrm{Yb}_{0.26} \mathrm{~B}_{12}$ shows a logarithmic divergence of the form $C_{\mathrm{mag}} / T \sim-\ln T$ at $T<4 \mathrm{~K}$, which may be attributed to the quantum critical regime, and it is suppressed by strong external magnetic field. The Schottky anomaly of the magnetic contribution to specific heat in $\operatorname{Tm}_{1-x} \mathrm{Yb}_{x} \mathrm{~B}_{12}$ has been established and analyzed in detail.
\end{abstract}

PACS numbers: $75.40 .-\mathrm{s}$

\section{Introduction}

Quantum criticality and the associated magnetic quantum phases of heavy fermion metals on one side and the metal-insulator transition (MIT) in strongly interacting electron systems on other side are of extensive current interest $[1,2]$. Moreover, the picture of a magnetic metal transformation into paramagnetic insulator state has been subjected to experimental testing in recent years. In particular, it was found very recently [3] that in the family of solid solutions $\mathrm{Tm}_{1-x} \mathrm{Yb}_{x} \mathrm{~B}_{12}$ the substitution of $\mathrm{Tm}$ by $\mathrm{Yb}$ causes a transition from antiferromagnetic metal $(\mathrm{AF}) \mathrm{TmB}_{12}\left(T_{\mathrm{N}} \approx 3.2 \mathrm{~K}\right)$ through the quantum critical point $(\mathrm{QCP})$ with $T_{\mathrm{N}} \approx 0$ at $x_{\mathrm{C}} \approx 0.3$ to the so-called Kondo insulator state in $\mathrm{YbB}_{12}$. Additionally, the decreased dimension of the magnetic excitation spectrum was revealed both in the case of $\mathrm{YbB}_{12}$ narrow-gap semiconductor [4] and in the well-known and most extensively studied system with the AF-type QCP - $\mathrm{CeCu}_{5.9} \mathrm{Au}_{0.1}$ [5]. In analogy with the $\mathrm{CeCu}_{6-x} \mathrm{Au}_{x}$ system, it is interesting to investigate in detail the behavior of specific heat $C_{p}(T)$ for $\mathrm{Tm}_{1-x} \mathrm{Yb}_{x} \mathrm{~B}_{12}$ compounds in a wide vicinity of $\mathrm{AF}$ near QCP $x_{\mathrm{C}} \approx 0.3$ and within MIT. Since the magnetic field is a crucial parameter in the quantum critical region [1, 5], it seems reasonable also to perform $C_{p}(T)$ measurements in high magnetic fields.

\section{Experimental}

In this work the behavior of specific heat of $\mathrm{Tm}_{1-x} \mathrm{Yb}_{x} \mathrm{~B}_{12}$ solid solutions has been studied on high

\footnotetext{
* corresponding author; e-mail: nes@lt.gpi.ru
}

quality single crystals with $0 \leq x \leq 0.8$ within the temperature range of $1.9-300 \mathrm{~K}$ in magnetic field up to $9 \mathrm{~T}$ in a PPMS-9 (Quantum Design). For comparison the non-magnetic reference compound $\mathrm{LuB}_{12}$ was also investigated in present study. The $\mathrm{RB}_{12}$ single crystals were grown by vertical crucible-free induction zone melting in an inert gas atmosphere [6].

\section{Results and discussion}

Figures 1 and 2 show the temperature dependences of specific heat for the antiferromagnet $\mathrm{TmB}_{12}$, $\mathrm{Tm}_{0.69} \mathrm{Yb}_{0.31} \mathrm{~B}_{12}$ compound in vicinity of $\mathrm{QCP}$, non-magnetic reference dodecaboride $\mathrm{LuB}_{12}$ (Fig. 1), and for paramagnetic solid solutions $\mathrm{Tm}_{1-x} \mathrm{Yb}_{x} \mathrm{~B}_{12}$ with $x=$ $0.23,0.37$ and 0.72 (Fig. 2). The heat capacity of $\mathrm{LuB}_{12}$ was measured to estimate the Sommerfeld $C_{\mathrm{e}}(T)$ and lattice $C_{\mathrm{ph}}(T)$ components in the specific heat of $\mathrm{Tm}_{1-x} \mathrm{Yb}_{x} \mathrm{~B}_{12}$ and to extract the magnetic contribution $C_{\text {mag }}(T)=C_{p}(T)-C_{\mathrm{e}}(T)-C_{\mathrm{ph}}(T)$. Figure 3 presents, for example, the temperature dependences $C_{\text {mag }}(T)$ of $\mathrm{Tm}_{0.74} \mathrm{Yb}_{0.26} \mathrm{~B}_{12}$ obtained at different values of external magnetic field $\mu_{0} H=0,3,6$ and $9 \mathrm{~T}$. The data presentation $C_{\mathrm{mag}} / T=f(\ln T)$ allows us to conclude in favor of the logarithmic divergence of the renormalized Sommerfeld coefficient at low temperatures, which is typical for systems at QCP [7], and it is attributed usually to the dramatic renormalization of the quasiparticles' effective mass and to the issue of a non-Fermi-liquid behavior of heat capacity.

It should be noted also that the magnetic field $\mu_{0} H \geq$ $3 \mathrm{~T}$ suppresses completely the quantum critical regime (Fig. 3) and that at low temperatures the $C_{\text {mag }}(T)$ curves demonstrate the Schottky anomaly of specific heat. The 


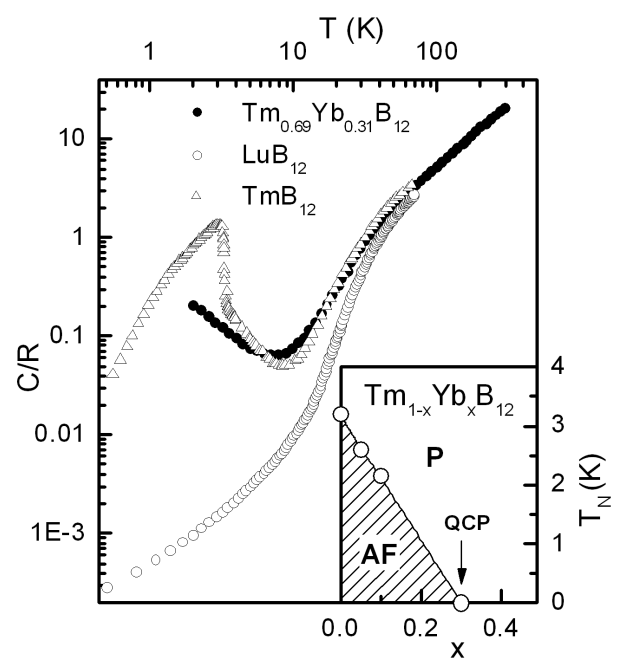

Fig. 1. Dependences of specific heat vs. temperature for dodecaborides $\operatorname{Tm}_{1-x} \mathrm{Yb}_{x} \mathrm{~B}_{12}$ with $x=0$ and 0.31 and for non-magnetic reference dodecaboride $\mathrm{LuB}_{12}$. The inset presents the $T-x$ phase diagram.

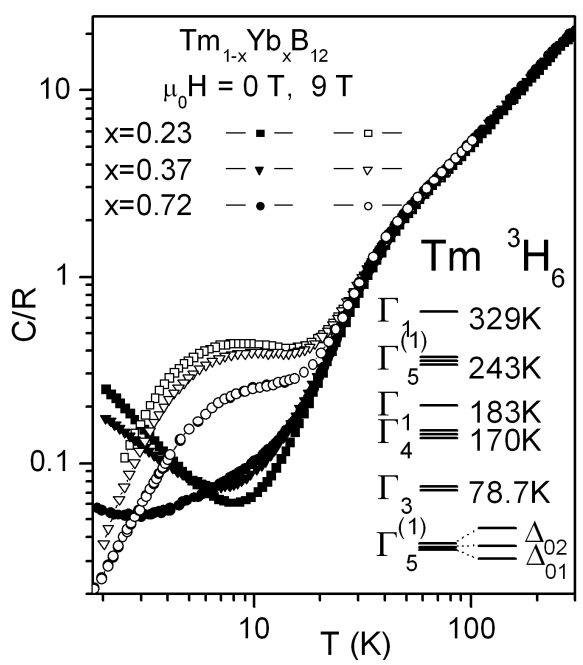

Fig. 2. Dependences of specific heat vs. temperature for dodecaborides $\mathrm{Tm}_{1-x} \mathrm{Yb}_{x} \mathrm{~B}_{12}$ with $x=0.23,0.37$ and 0.72 at $H=0$ and $9 \mathrm{~T}$. In the inset the scheme of crystal field splitting of the $\mathrm{Tm}^{3+}{ }^{3} H_{6}$ state is presented.

detailed numerical analysis of the crystal electric field and the Zeeman splitting features of the magnetic contribution allows us both to confirm the scheme of the $\mathrm{Tm}^{3+}{ }^{3} H_{6}$ state splitting (inset in Fig. 2), and to estimate the effective magnetic moments $\left(\mu_{\mathrm{eff}} \approx 2.82 \mu_{\mathrm{B}}\right.$ and $4.8 \mu_{\mathrm{B}}$, correspondingly) for the states $\Delta_{01}$ and $\Delta_{02}$ of the $\Gamma_{5}^{(1)}$-triplet of $\mathrm{Tm}^{3+}$ (see inset in Fig. 3). The results of $C_{\mathrm{mag}}(T)$ and entropy studies indicate that the Tm subsystem should be considered as a decisive factor

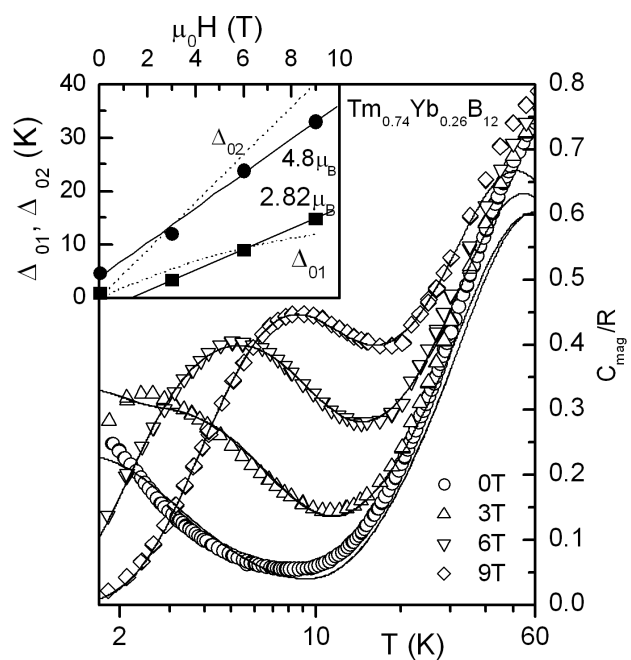

Fig. 3. Temperature dependences of the magnetic contribution $C_{\mathrm{mag}}(T)$ to specific heat of $\mathrm{Tm}_{0.74} \mathrm{Yb}_{0.26} \mathrm{~B}_{12}$ in external magnetic field up to $9 \mathrm{~T}$. Inset shows the Zeeman splitting $\left(\Delta_{01}, \Delta_{02}\right)$ of the $\Gamma_{5}^{(1)}$ triplet of the $\mathrm{Tm}^{3+}{ }^{3} H_{6}$ state in magnetic field.

in the formation of features of the thermal properties in the $\mathrm{Tm}_{1-x} \mathrm{Yb}_{x} \mathrm{~B}_{12}$ strongly correlated electron system.

\section{Acknowledgments}

This work was supported by the program of the Branch of Physical Sciences of RAS, by the RAS and SAS cooperation program, and by research grants VVCE-0058, APVV-0346-07, VEGA-0148 and RFBR 10-02-00998-a.

\section{References}

[1] Q. Si, S. Rabello, K. Ingersent, J.L. Smith, Nature 413, 804 (2001).

[2] A. Akbari, P. Thalmeier, P. Fulde, Phys. Rev. Lett. 102, 106402 (2009).

[3] N.E. Sluchanko, A.V. Bogach, V.V. Glushkov, S.V. Demishev, K.S. Lyubshov, D.N. Sluchanko, A.V. Levchenko, A.B. Dukhnenko, V.B. Filipov, S. Gabani, K. Flachbart, JETP Lett. 89, 256 (2009).

[4] K.S. Nemkovski, J.M. Mignot, P.A. Alekseev, A.S. Ivanov, E.V. Nefedova, A.V. Rybina, L.P. Regnault, F. Iga, T. Takabatake, Phys. Rev. Lett. 99, 137204 (2007).

[5] O. Stockert, H. von Lohneysen, A. Rosch, N. Pyka, M. Loewenhaupt, Phys. Rev. Lett. 80, 5627 (1998).

[6] Yu. Paderno, V. Filippov, N. Shitsevalova, AIP Conf. Proc. 230, 460 (1991).

[7] H. von Lohneysen, J. Phys., Condens. Matter 8, 4889 (1996). 